\title{
Drug Use Evaluation of Letrozole in Breast Cancer Patients at Regional Cancer Hospitals in Thailand
}

\author{
Chaninun Ketkaew*, Niyada Kiatying-Angsulee
}

\begin{abstract}
Background: Medication policy development in Thailand is continually promoting rational drug use. Letrozole, an endocrine therapy drug, is usually prescribed for post-menopausal status early and advanced stage breast cancer. After Ministry of Public Health announced Letrozole as compulsory licensed drug in 2009, more breast cancer patients can access to this drug at low cost especially those within universal coverage schemes. To ensure that Letrozole is rationally prescribed, the drug utilization study was conducted. Objectives: The aim of this study was to describe the appropriate use of Letrozole in breast cancer and the relationship between appropriate use and health benefit schemes. Materials and Methods: A retrospective study to evaluate use of Letrozole in breast cancer patients was performed for six months between January - June 2010 in seven regional cancer hospitals, Thailand. All prescriptions of Letrozole were identified from pharmacy dispensing databases and prescription papers. A medical record review was also performed to evaluate appropriate use referring to the drug use evaluation criteria. The approved criterion of this study was referred from the guideline of Thai National Formulary version 2010. Results: There were 681 prescriptions of Letrozole for 254 breast cancer patients with an average age of $58.6 \pm 10.0$ years. The patients in universal coverage scheme (UCS), civil servant medication benefit scheme (CSMBS) and social security scheme (SSS) were $77.7 \%, 18.5 \%$ and $8.7 \%$ respectively. $10.6 \%$ were prescribed Letrozole for the first time. Letrozole were prescribed by oncologists $(\mathbf{8 2 . 8 \%})$. The average number of tablets per prescription was $58 \pm 10$. Calcium supplements were prescribed concomitant with Letrozole for 19.4\%. To assess drug use evaluation criteria, 45 prescriptions were excluded because of uncompleted clinical data, 636 prescriptions were evaluated. The study showed 86 prescriptions $(13.5 \%)$ with inappropriate use including $6(0.9 \%)$ not prescribed for estrogen receptor (ER) and/or progesterone receptor (PR) positive, 31 (4.9\%) not prescribed for post-menopausal and $49(7.7 \%)$ not prescribed for an appropriate duration. Appropriate use percentages in different health benefit schemes were similar, 85.7\% of CSMBS, 86.4\% of SSS and 86.7\% of UCS. The relationship between health benefit scheme and appropriate use of Letrozole was not significantly different, $\chi 2(2, N=636)=0.081, p>0.05$. Conclusions: The study showed inappropriate use in breast cancer patients because of non-compliance with duration, menopausal status and hormone receptor requirements. To prescribe appropriate indication did not referred to the appropriate practice along the treatment. Drug use evaluation proved very useful for detecting the sign of inappropriate use and allows immediate feedback to the stakeholder for developing medication policy in the future. Importantly, there was no significantly difference in appropriate use of Letrozole across health benefit schemes.
\end{abstract}

Keywords: Rational drug use - drug use evaluation - breast cancer - letrozole

Asian Pac J Cancer Prev, 16 (14), 6055-6059

\section{Introduction}

World Health Organization (WHO) defines rational use of drugs as patients receive medication appropriate to their clinical needs, in doses that meet their own individual requirements, for an adequate period of time, and at the lowest cost to them and their community(World Health Organization, 2002). Drug use evaluation (DUE) is a tool for monitoring how rationally drugs are prescribed. Many drugs use problems that suggested a need for DUE included several choices of therapy, a pattern of diversity among prescribers, high number of adverse drug reactions, sign of treatment failures, excessive number of nonformulary drugs used and use of high cost drugs where less expensive alternatives exist. The DUE composed of 4 process as, first define appropriate drug use by establishing approved criteria, second audit criteria against what is being prescribed, third provide feedback to prescribers on all identified problems, and finally monitor to see if criteria are followed and prescribing improved(Health, 2007). Many studies focused on evaluated use of cancer drug especially targeted therapy drugs and chemotherapy 
Chaninun Ketkaew and Niyada Kiatying-Angsulee

drugs because of its expensive and high risk in adverse drug reaction (Delpeuch, Leveque, Rob, \& Bergerat, 2011; Gulam Muhammad Khan, 2013; Joerger et al., 2014; Krzyzanowska, 2013; Lerose, Musto, Aieta, Papa, \& Tartarone, 2012; Pasqualetti et al., 2012; Siddiqua A., 2014; Tartarone, Lerose, \& Aieta, 2012; Wang et al., 2013). Few studies focused on evaluation of endocrine therapy in breast cancer, for example, Kahan N.R. et al. showed $5.8 \%$ off-label use of Tamoxifen in Isael(Kahan, Waitman, Blackman, \& Vardy, 2010). This study aimed to describe the appropriate use of Letrozole referred to the treatment guideline by Thai National Formulary (TNF) version 2010 (National List of Essential Medicine Development Subcomittee, 2010; National List of Essential Medicines, 2009) and describe the pattern of use between three health benefit schemes. Letrozole is endocrine therapy drug for breast cancer as inhibit hormone action and stop tumor cell growth (Barnadas et al., 2011; Cohen, Johnson, Justice, \& Pazdur, 2011; Lee, Armstrong, \& Wardley, 2012; Shaw \& Ellis, 2002). It was launched in Thailand more than ten years ago but not listed in the National Essential Drug List until 2010. Because of expensive drug, sale price of original drug was 256 Baht/tablet in 2009 (7 - 8 US \$/tablet, 1 US\$=35 Baht) and major health schemes was not covered. In 2010, The Ministry of Public Health announced Letrozole as compulsory licensed drugs that was centrally procured and distributed by The Government Pharmaceutical Organization (GPO). After that Letrozole was listed in the National Essential Drug List of Thailand 2010 in J-2 category and its sale price was only 7 Baht/ tablet (0.20 US\$/tablet). To ensure that breast cancer patients can appropriately access to Letrozole, DUE process should be performed.

\section{Materials and Methods}

Retrospective research was performed at seven regional cancer hospitals in Chonburi, Lopburi, Lumpang, Pathumthani, Ubonrachathani, Udonthani and Suratthani. Regional cancer hospitals were referral hospital for cancer. All hospitals should be met four system criteria.

1) The hospitals were tertiary care in cancer treatment due to the National Health Security Organization (NHSO) guideline.

2) The hospital must be registered in NHSO hospital lists

3) The specialists of each hospital must be registered as Letrozole's prescriber

4) The patients of each hospital must be registered as breast cancer patients

All prescriptions of Letrozole between January - June 2010 were collected from prescription paper and pharmacy dispensing database. The information obtained from prescriptions were prescriptions' date, hospital number $(\mathrm{HN})$, ages, health benefit schemes, specialty of physician, dose and administration, amount of Letrozole tablet and calcium supplement prescribing data. Those data were recorded in data collection form by trained pharmacists. The medical record of patients were reviewed for the clinical characteristic and compare with DUE criteria.

The establishing approved criterion of this study was referred from DUE of Letrozole in the TNF guideline version 2010 (National List of Essential Medicine Development Subcomittee, 2010). The DUE criteria included:

1) Indication: Letrozole should be prescribed for breast cancer

- Advance breast cancer

- Adjuvant therapy as switching therapy in early stage breast cancer in two choices

a. Start with Tamoxifen for $2-3$ years and follow by Letrozole until 5 years total of hormone therapy

b. Start with Letrozole for 2 years and follow by Tamoxifen for 3 years until 5 years of hormone therapy

2) Hormone receptor status: Letrozole should be prescribed in Estrogen receptor positive (ER) and/or Progesterone receptor positive (PR).

3) Menopausal status: Letrozole should be prescribed only in post-menopausal defined as

- Age more than 60 years old

- Ovarian ablation

- Post-menopausal occurred 1 year before breast cancer diagnosed and confirm by follicle stimulating hormone (FSH) and luteinizing hormone (LH)

4) Physical status: Letrozole should not be prescribed in terminal illness patients

5) Dose: Appropriate dose of Letrozole was $2.5 \mathrm{mg}$. tablet oral once daily after meal

6) Duration: Depend on stage on breast cancer

- Advance breast cancer: Letrozole should be prescribed until disease progress or poorly respond

- Early stage breast cancer: Letrozole should be prescribed continuity not more than 2 years

The information obtained from the medical record included indication of use, duration of use, ER/PR receptor status, menstrual status, physical status and adverse drug reactions. Those data were recorded in DUE criteria check list by trained pharmacists. The SPSS/Win version 17.0 was used to analyze the data. The descriptive statistic was used to describe demographic characteristic of prescriptions of Letrozole, clinical data of patients and result in appropriate use. Chi square test of independence was used to analyze the relation between appropriate use and health benefit schemes.

\section{Results}

Demographic data of the prescription of Letrozole has been shown in Table 1 . There were 681 prescriptions of Letrozole for 254 patients. The average age of patients was $58.6 \pm 10.0$ years old. Patients were in Universal Coverage Scheme (UCS) (77.7\%), Civil Servant of Medication Benefit Scheme (CSMBS) (18.5\%) and Social Security Scheme (SSS) (8.7\%). 10.6\% were prescribed of Letrozole for the first time. Letrozole were prescribed by oncologists $(82.8 \%)$, radiologists $(13.7 \%)$ and surgeons $(3.5 \%)$. The average amount of tablets per prescription was $58 \pm$ 10 tablets. $100.0 \%$ of prescriptions were appropriately dose prescribed. $19.4 \%$ of Letrozole prescriptions were prescribed with calcium supplement.

The clinical data of breast cancer patients obtained from medical record has been shown in Table 2. The 
clinical criteria for evaluate appropriate use of Letrozole composed of diagnosis, hormone receptor, menstrual status, physical status, recommend dose and adverse reaction. 177 prescriptions $(25.9 \%)$ were prescribed in advance stage breast cancer and 284 prescriptions (74.0\%) in early stage. 654 prescriptions $(96.0 \%)$ were prescribed in ER and/or PR positive. Menopausal status was defined as post-menopause and pre-menopause, 620 prescriptions $(91.0 \%)$ were prescribed in post-menopause. All prescriptions of Letrozole were not prescribed for patients in terminal illness. Common adverse drug reactions were reported in 11 prescriptions $(1.60 \%)$ including bone pain, flushing and edema.

45 from 681 prescriptions were excluded from this study because lack of clinical data such as hormone receptor status and/or menstrual status. 636 prescriptions of Letrozole were analyzed with 1 - 5 DUE criteria respectively.

Criteria 1: Letrozole must be rationally prescribed in ER (+) and/or PR (+) (ER(+)/PR(+), ER(+)/PR(-) and ER()$/ \mathrm{PR}(+))$. Mechanism of Letrozole is inhibiting of breast tumor growth by inhibiting biosynthesis of hormone, so high density of hormone receptor show better efficacy. 6 from 636 prescriptions $(0.9 \%)$ were not appropriately prescribed for ER and/or PR positive. 630 appropriated prescriptions were continually evaluated in next criteria.

Criteria 2: Letrozole must be rationally prescribed in post-menopausal breast cancer patients. Post-menopausal can be identified by 3 criteria: 1) Age more than 60 years old, 2) under ovarian ablation 3) Post-menopausal occurred for 1 year before breast cancer diagnosed and confirm by FSH and LH. Pre-menopausal patients show estrogen effect that stimulates tumor cell growth. There were 31 prescriptions (4.9\%) of Letrozole prescribed in pre-menopausal. Those were evaluated as inappropriate use. 599 prescriptions were continually evaluated in next criteria.

Criteria 3: Approve indication of Letrozole was in advance breast cancer and adjuvant therapy in early stage breast cancer. All 599 prescriptions were appropriated indication.

Criteria 4: The appropriate dose of Letrozole was fixed as $2.5 \mathrm{mg}$ tablet orally once a day. All 599 prescriptions were prescribed in appropriate dose.

Criteria 5: The appropriate duration of letrozole were defined in line with the indication as follow
-Advance breast cancer: Letrozole can be prescribed continually until the disease progress.

Table 1. Demographic Characteristic of the Prescriptions of Letrozole

\begin{tabular}{lr}
\hline Demographic Data & Result (\%) \\
\hline Number of prescriptions & 681 \\
Number of patients & 254 \\
Age of patients (years) & $58.6 \pm 10.0$ \\
Health benefit schemes & \\
Civil Servant of Medication Benefit Scheme (CSMBS) (\%) \\
\multicolumn{1}{l}{ Universal Coverage Scheme (UCS) (\%) } & $126(18.5)$ \\
Social Security Scheme (SSS) (\%) & $488(77.7)$ \\
Other & $59(8.7)$ \\
First prescribed & $8(1.2)$ \\
Yes (\%) & $72(10.6)$ \\
No (\%) & $609(89.4)$ \\
Prescriber & \\
Oncologist (\%) & $564(82.8)$ \\
Radiologist (\%) & $93(13.7)$ \\
Surgeon (\%) & $24(3.5)$ \\
Average amount of tablet per prescription & \\
Mean \pm SD (Tablet) & $57.8 \pm 10.1$ \\
Recommended dose (2.5 mg Tablet oral daily) & $681(100.0)$ \\
Prescribed Calcium Supplement & $132(19.4)$ \\
\hline
\end{tabular}

Table 2. The Clinical Data of Breast Cancer Patients Obtained from Medical Record Charges

\begin{tabular}{lc}
\hline Clinical data requirement & Number of prescriptions (\%) \\
\hline Diagnosis & $177(25.9)$ \\
Advanced breast cancer & $284(41.7)$ \\
Early breast cancer: Adjuvant therapy (Start with Tamoxifen) \\
\multicolumn{2}{c}{$220(32.3)$} \\
Early breast cancer: Adjuvant therapy (Start with Letrozole) \\
Hormone receptor: Estrogen (ER), Progesterone (PR) \\
ER (+)/PR (+) & $423(62.1)$ \\
ER (+)/PR (-) & $204(30.0)$ \\
ER (-)/PR (+) & $27(4.0)$ \\
ER (-)/PR (-) & $6(0.9)$ \\
N/A & $21(3.1)$ \\
Menopausal status & \\
Post-menopause & $620(91.4)$ \\
Pre-menopause & $31(4.6)$ \\
N/A & $30(4.4)$ \\
Terminal illness & \\
No & $681(100.0)$ \\
Adverse event occurred & $11(1.6)$ \\
\hline
\end{tabular}

Table 3. Drug Use Evaluation Result of Letrozole Referred to the Guideline by TNF Version 2010

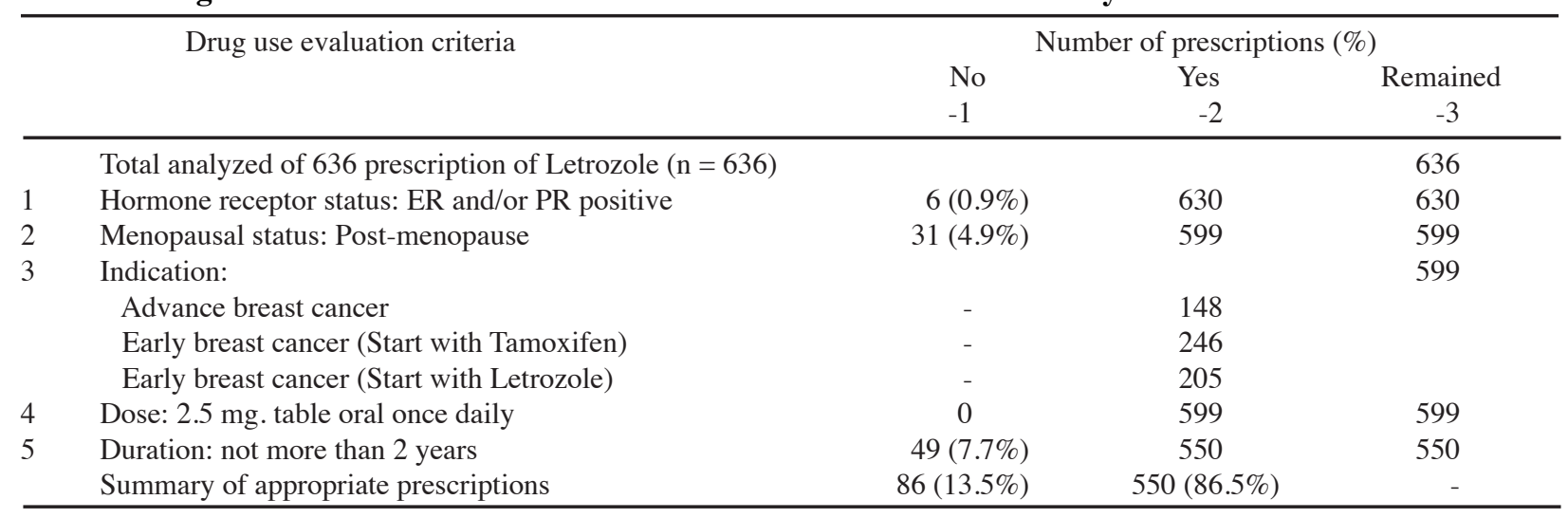


Chaninun Ketkaew and Niyada Kiatying-Angsulee

Table 4. Relation Analysis Evaluation Result of Appropriated use of Letrozole in Different Health Benefit Schemes

\begin{tabular}{lrrr}
\hline Health Benefit & \multicolumn{2}{c}{ Number of prescriptions (\%) } & \\
Schemes & Appropriate & Inappropriate & Total \\
\hline CSMBS & $108(85.7 \%)$ & $18(14.3 \%)$ & 126 \\
SSS & $51(86.4 \%)$ & $8(13.6 \%)$ & 59 \\
UCS & $391(86.7 \%)$ & $60(13.3 \%)$ & 451 \\
Total & $550(86.5 \%)$ & $86(13.5 \%)$ & 636 \\
\hline
\end{tabular}

$\mathrm{X}^{2}=0.081$; Pearson Chi-Square $\mathrm{P}=0.960$

-Adjuvant therapy in early stage breast cancer: Letrozole can be prescribed before and after Tamoxifen as switching therapy but not more than 2 years.

For these criteria, 49 prescriptions $(7.7 \%)$ were inappropriate duration of prescribed.

Finally there were $86(13.52 \%)$ inappropriate prescriptions of Letrozole, and $550(86.48 \%)$ appropriated prescriptions from totally 636 prescriptions.

To describe appropriate use in different health benefit schemes, the study showed similarly percentage of appropriate prescriptions. The prescription of Letrozole in CSMBS was appropriated as $85.7 \%$, SSS was $86.4 \%$ and UCS was $86.7 \%$. A chi-square test of independence examined the relationship between health benefit scheme (CSMBS, SSS and UCS) and appropriate use of Letrozole (Appropriate, Inappropriate). The relationship between these variables was not statistically significant, $\chi^{2}(2$, $\mathrm{N}=636)=0.081, \mathrm{p}>0.05$.

\section{Discussion}

This study show inappropriate use of Letrozole in 3 criteria including inappropriate duration $(7.7 \%)$, inappropriate menopausal status $(4.9 \%)$ and inappropriate hormone receptor $(0.9 \%)$. TNF guideline 2010 indicates the total duration of endocrine therapy in early stage breast cancer as sequential treatment (start with Tamoxifen for 3 years then followed by Letrozole for 2 years /strat with Letrozole for 2 years then followed by Tamoxifen for 3 years) not more than 5 years. That was consistent with the previous studies included BIG 1-98 trials and others (Gelmon, 2007; Goss, 2007; Regan et al., 2011). However prescribing Tamoxifen for 5 years in early breast cancer patients still practically, those patients always continue with Letrozole for 2 years. That caused total duration of endocrine therapy was more than 5 years and be evaluated as inappropriate use. Other reason for inappropriate duration of Letrozole related to the National List of Essential Drug that already contained Tamoxifen for a long time and it always be drug of choices, although early study that show the significant benefit of starting Letrozole in early breast cancer because of its good efficacy and less adverse reaction (Bundred, 2006; Scott \& Keam, 2006). Furthermore, some patients in this study started endocrine treatment before TNF guideline was established, by previous treatment may be based on the previous guideline and was not consistence with the TNF guideline. Some patients may be first prescribed other aromatase inhibitor (Nabholtz et al., 2009) (Exemestane or Anastrozole) before shifted to Letrozole. The total duration aromatase inhibitor were accumulated, however the study in Thailand showed not significant respond after aromatase inhibitor was shifted(Aphinives, Vachirodom, Thanapaisal, Rangsrikajee, \& Somintara, 2014). Letrozole should be prescribed only in hormone receptor positive and postmenopause (Keating, 2009; Mann et al., 2005; Scott \& Keam, 2006; Yadav, Sharma, Patel, Ghoshal, \& Kapoor, 2007) because of avoiding estrogenic effect from ovary. For $4.9 \%$ inappropriate use in premenopausal, these were found in early stage breast cancer who were prescribed Tamoxifen for 5 years and switched to Letrozole while they still premenopausal. For $0.9 \%$ inappropriate use in hormone receptor negative (ER- and PR-), some pathology included ER/PR always be reviewed when the patients referred. Prescribing Letrozole in ER(-)/PR(-) were found.

The breast cancer patients who visited at cancer hospital were under 3 main health benefit schemes; universal coverage scheme (UCS), civil servant medication benefit scheme (CSMBS) and social security scheme (SSS). $7.80 \%$ of Thai people are in CSMBS, $15.46 \%$ in SSS and $75.70 \%$ in UCS(Jindapol N., 2014). Some study showed trend of inappropriate use and high consume of medication cost in CSMBS(Jindapol N., 2014). This study aimed to investigate the different in appropriate use of Letrozole in any schemes. Although the appropriate use in UCS was related with the reimbursement condition more than other schemes, but the result showed not significant in appropriate use of Letrozole in different health benefit schemes $(\mathrm{p}=0.081)$.

In conclusion, Letrozole has a major role as endocrine therapy in early stage and advanced breast cancer. To evaluated how appropriate use of Letrozole in breast cancer patients, the study showed $13.52 \%$ of inappropriate use because of duration, menopausal status and hormone receptor. To prescribe appropriate indication did not referred to the appropriate practice along the treatment. Drug use evaluation was very useful for detecting the sign of inappropriate use and immediately feedback to the stakeholder for developing medication policy in the future. Furthermore there was not significantly different in appropriate use of Letrozole in any health benefit schemes.

Limitations: as the referral cancer hospital, majority of patients were referred and previous treated with Letrozole from other hospital. Some missing clinical data and unaccountable number of Letrozole were excluded as $6.61 \%$ (45 from 681 prescriptions).

\section{Acknowledgements}

The study has been completed successfully with excellent support from the director and head of pharmacy department of regional cancer hospitals. The study had been supported from Thai Drug Watch and Chulalongkorn Graduate School Thesis Grant, 2012.

\section{References}

Aphinive P, Vachirodom D, Thanapaisal C, Rangsrikajee D Somintara O (2014). Effects of switching from anastrozole to letrozole, due to reimbursement policy, on the outcome of breast cancer therapy. Breast Cancer, 6, 145-150. 
Barnadas A, Estevez LG, Lluch-Hernandez A, RodriguezSanchez C, Sanchez-Rovira P (2011). An overview of letrozole in postmenopausal women with hormoneresponsive breast cancer. Adv Ther, 28, 1045-58.

Bundred NJ (2006). Letrozole: a well-tolerated and effective treatment for breast cancer. Womens Health, 2, 673-85.

Cohen MH, Johnson JR, Justice R, Pazdur R (2011). Approval summary: letrozole (Femara(R) tablets) for adjuvant and extended adjuvant postmenopausal breast cancer treatment: conversion of accelerated to full approval. Oncologist, 16 1762-70.

Delpeuch A, Leveque D, Rob L, Bergerat JP (2011). Off-label use of oxaliplatin in patients with metastatic breast cancer. Anticancer Res, 31, 1765-7.

Gelmon K (2007). Prescribing extended adjuvant letrozole. Breast, 16, 446-55.

Goss PE (2007). Letrozole in the extended adjuvant setting: MA.17. Breast Cancer Res Treat, 105, 45-53.

Gulam Muhammad Khan RKT, Dewashish Singh Adhikari, Muna Rajbhandari, et al (2013). Evaluation of cancer prevalence and cytotoxic medication prescribing in central region of Nepal. Kathmandu University, J Sci Eng Technol, 9, 189-99.

Health MSf (2007). Drug use evaluation. in the \& R. P. M. P. Program (Eds.), Drug and therapeutics committee. Training Course (pp. 1-9). United States: Management Sciences for Health.

Jindapol N., K. P. e. a. (2014). Crisis of health insurance under Thai civil servant medical benefit scheme (CSMBS). Srinagarin Med J, 9, 199-206.

Joerger M, Schaer-Thuer C, Koeberle D, et al (2014). Off-label use of anticancer drugs in eastern Switzerland: a populationbased prospective cohort study. Eur J Clin Pharmacol, 70, 719-25.

Kahan NR., Waitman DA, Blackman S, Vardy DA(2010). Drug use evaluation of tamoxifen focusing on off-label use in a managed care population in Israel. J Manag Care Pharm, 16, 355-9.

Keating GM(2009). Letrozole: a review of its use in the treatment of postmenopausal women with hormone-responsive early breast cancer. Drugs, 69, 1681-705.

Krzyzanowska MK (2013). Off-label use of cancer drugs: a benchmark is established. J Clin Oncol, 31, 1125-7.

Lee R J, Armstrong AC, Wardley AM (2012). Letrozole: advancing hormone therapy in breast cancer. Womens Health (Lond Engl), 8, 611-8.

Lerose R, Musto P, Aieta M, Papa C, Tartarone A (2012). Offlabel use of anti-cancer drugs between clinical practice and research: the Italian experience. Eur J Clin Pharmacol, 68, 505-12.

Mann BS, Johnson JR, Kelly R, et al (2005). Letrozole in the extended adjuvant treatment of postmenopausal women with history of early-stage breast cancer who have completed 5 years of adjuvant tamoxifen. Clin Cancer Res, 11, 5671-7.

Nabholtz JM, Mouret-Reynier MA, Durando X, Van Praagh I, Al-Sukhun S, Ferriere JP, Chollet P (2009). Comparative review of anastrozole, letrozole and exemestane in the management of early breast cancer. Expert Opin Pharmacother, 10, 1435-47.

National List of Essential Medicine Development Subcomittee. (2010). Thai national formulary: special access medicines of national list of essential medicines, [Epub ahead of print].

National List of Essential Medicines. (2009). National list of essential medicines Thailand 2009. Retrieved from http:// drug.fda.moph.go.th:81/nlem.in.th/node/10351.

Pasqualetti G, Ricci S, Boni G, et al (2012). Off-label use of sunitinib in patients with advanced, epithelial thyroid cancer: a retrospective analysis. Recent Pat Endocr Metab Immune Drug Discov, 6, 171-6.

Regan MM, Neven P, Giobbie-Hurder A, et al (2011). Assessment of letrozole and tamoxifen alone and in sequence for postmenopausal women with steroid hormone receptorpositive breast cancer: the BIG 1-98 randomised clinical trial at 8.1 years median follow-up. Lancet Oncol, 12, 1101-8.

Scott LJ, Keam SJ (2006). Letrozole : in postmenopausal hormone-responsive early-stage breast cancer. Drugs, 66, 353-62.

Shaw HS, Ellis MJ (2002). Letrozole in the treatment of breast cancer. Expert Opin Pharmacother, 3, 607-17.

Siddiqua A, Tabassum N, Firdous S, Tabassum K (2014). Drug utilization evaluation of anti cancer drugs. Am J Pharmtech Res, 4, 690-702.

Tartarone A, Lerose R, Aieta M (2012). Reflections about offlabel use of anticancer drugs. Ther Drug Monit, 34, 604.

Wang W, Zhu M, Guo D, et al (2013). Off-label and off-NCCN guidelines uses of antineoplastic drugs in China. Iran $J$ Public Health, 42, 472-9.

World Health Organization (2002). Promoting rational use of medicine: core component. Retrieved from http://apps.who. int/medicinedocs/pdf/h3011e/h3011e.pdf.

Yadav BS, Sharma SC, Patel FD, Ghoshal S, Kapoor V (2007). Results of letrozole in postmenopausal women after tamoxifen treatment for advanced breast cancer. $J$ Cancer Res Ther, 3, 71-4. 